\title{
An International registry on Autoinflammatory diseases: the Eurofever experience
}

\author{
Natasa Toplak, ${ }^{1}$ Joost Frenkel, ${ }^{2}$ Seza Ozen, ${ }^{3}$ Helen J Lachmann, ${ }^{4}$ Patricia Woo, ${ }^{5}$ \\ Isabelle Koné-Paut, ${ }^{6}$ Fabrizio De Benedetti, ${ }^{7}$ Benedicte Neven, ${ }^{8}$ Michael Hofer, ${ }^{9}$ \\ Pavla Dolezalova, ${ }^{10}$ Jasmin Kümmerle-Deschner, ${ }^{11}$ Isabelle Touitou, ${ }^{12}$ \\ Veronique Hentgen, ${ }^{13}$ Anna Simon, ${ }^{14}$ Herman Girschick, ${ }^{15}$ Carlos Rose, ${ }^{16}$ \\ Carine Wouters, ${ }^{17}$ Richard Vesely, ${ }_{18}^{18}$ Juan Arostegui, ${ }^{19}$ Silvia Stojanov, ${ }^{20}$ Huri Ozgodan, ${ }^{21}$ \\ Alberto Martini, 22 Nicolino Ruperto, ${ }^{23}$ Marco Gattorno ${ }^{23}$ for the Paediatric Rheumatology \\ International Trials Organisation (PRINTO), Eurotraps and Eurofever Projects
}

\section{- Additional tables are published online only. To view the files please visit the journal online (http://ard.bmj.com/ content/71/7.toc). \\ For numbered affiliations see end of article \\ Correspondence to \\ Marco Gattorno, Pediatria II, Reumatologia, IRCCS Istituto \\ G Gaslini, Università di Genova \\ 5, 16147, Genova, Italy; \\ marcogattorno@ \\ ospedale-gaslini.ge.it}

Received 2 August 2011 Accepted 11 December 2011 Published Online First: 29 February 2012

\begin{abstract}
Objective To report on the demographic data from the first 18 months of enrollment to an international registry on autoinflammatory diseases in the context of the Eurofever project.

Methods A web-based registry collecting baseline and clinical information on autoinflammatory diseases and related conditions is available in the member area of the PRINTO web-site. Anonymised data were collected with standardised forms.
\end{abstract}

Results 1880 (M:F=916:964) individuals from 67 centers in 31 countries have been entered in the EUROFEVER registry. Most of the patients (1388; 74\%), reside in western Europe, $294(16 \%)$ in the eastern and southern Mediterranean region (Turkey, Israel, North Africa), 106 (6\%) in eastern Europe, 54 in Asia, 27 in South America and 11 in Australia. In total 1049 patients with a clinical diagnosis of a monogenic autoinflammatory diseases have been enrolled; genetic analysis was performed in 993 patients (95\%): 703 patients have genetically confirmed disease and 197 patients are heterozygous carriers of mutations in genes that are mutated in patients with recessively inherited autoinflammatory diseases. The median diagnosis delay was 7.3 years (range $0.3-76$ ), with a clear reduction in patients born after the identification of the first gene associated with autoinflammatory diseases in 1997. Conclusions A shared online registry for patients with autoinflammatory diseases is available and enrollment is ongoing. Currently, there are data available for analysis on clinical presentation, disease course, and response to treatment, and to perform large scale comparative studies between different conditions.

\section{INTRODUCTION}

The autoinflammatory diseases are a group of inflammatory conditions associated with abnormal regulation of innate immunity. ${ }^{1}$ The first molecular genetic characterisation of an autoinflammatory disease was in 1997, when two consortia independently identified the association between mutations of the MEFV (MEditerranean FeVer) gene and Familial Mediterranean fever (FMF). ${ }^{2}$ Over the last decade, a number of monogenic and multifactorial diseases have been identified or reclassified as autoinflammatory in aetiology ${ }^{14}$. With the sole exception of FMF, which is highly prevalent in some ethnicities (Turks, Arabs, non-Ashkenazi Jews and Armenians) $)^{5}$, the incidence of autoinflammatory diseases is low, ranging from estimated population frequencies of 1 to 3 per million for the cryopyrin associated periodic syndromes (CAPS) ${ }^{6}$ to less than 10 reported families for the most rare conditions, such as deficiency of IL-1 receptor antagonist (DIRA) or familial cold autoinflammatory syndrome 2 (FCAS2) associated with NLRP12 gene mutations. ${ }^{78}$

Our knowledge of autoinflammatory diseases, although expanding rapidly, is still very limited. Genotype-phenotype correlations and the potential influences of environmental and epigenetic factors are still largely unknown and, with the exception of CAPS and DIRA, their molecular pathogenesis remains elusive. Clinically, the rarity and phenotypic variability of most of these diseases means that delayed diagnosis is the norm. Even once a diagnosis has been reached we still struggle to provide patients and parents with definitive answers as to the best treatments available and their long term prognosis.

A major limitation to understanding these rare conditions has been the fragmentation of clinical experience with very few centers in any country caring for more than a handful of cases. In order to counteract this an international study, named EUROFEVER has been supported by the Executive Agency for Health and Consumers of European Union (EAHC, Project No2007332) ${ }^{9}$. The general aims of the project are: (i) to promote awareness and enhance early recognition of these diseases among the medical community; (ii) to provide clear and comprehensive information to families and patients affected by these conditions, (iii) to improve knowledge of the clinical presentation, response to treatment and long term complications of these disorders. The major practical part of the project was to set up an international webbased registry of autoinflammatory diseases. In this article we report on the demographic data of the patients enrolled during the first 18 months of the EUROFEVER registry. 


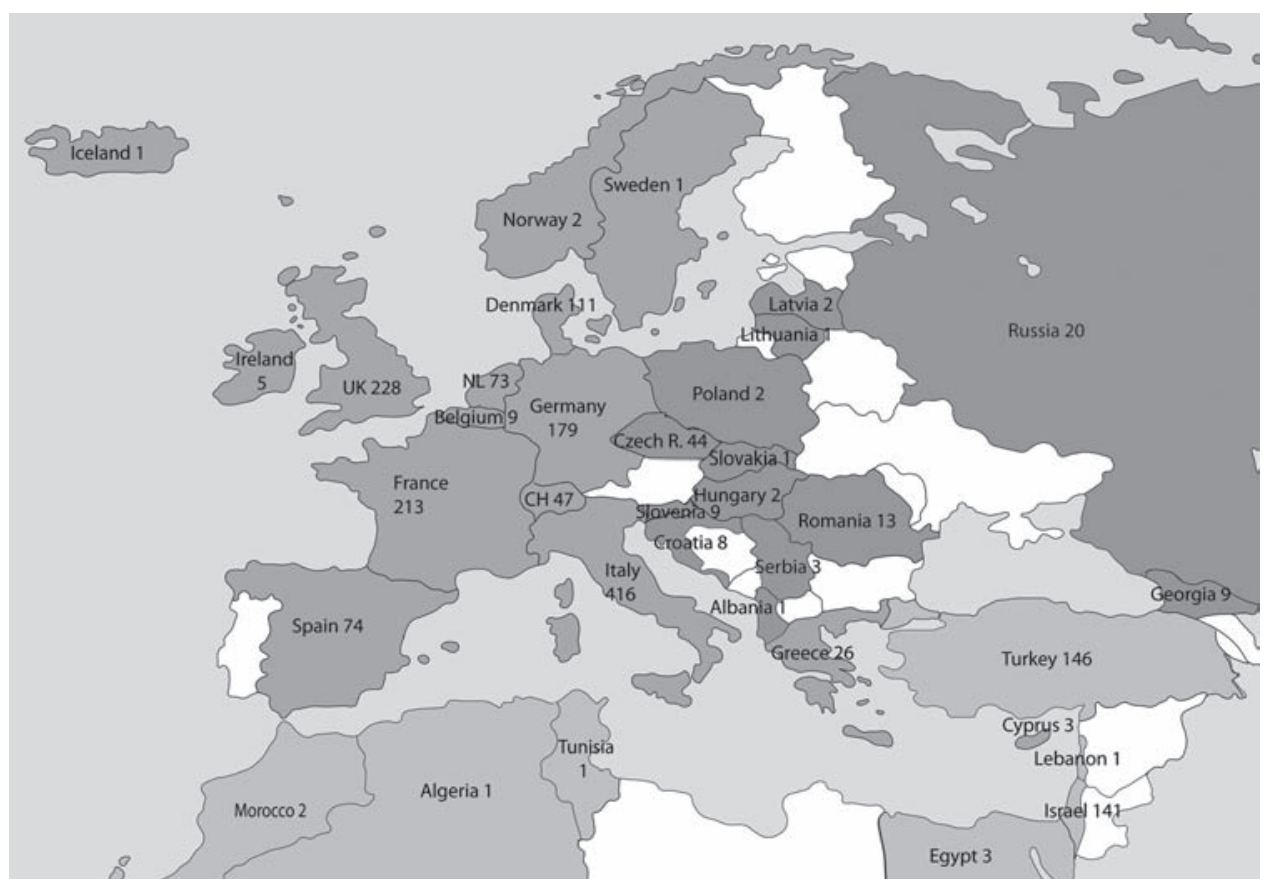

Figure 1 Distribution of patients according to the country of residency in Europe and the Mediterranean region. Other patients living in Saudi Arabia (33), Argentina (23), Australia (11), India (5), Oman (4), Kuwait (3), Guadalupe (2), Uruguay (1) and Martinique (1) are also reported.

Table 1 Demographic characteristics of patients enrolled in the registry in the first 18 months

\begin{tabular}{|c|c|c|c|c|c|c|c|c|}
\hline Disease & Patient number & $M / F$ & $\begin{array}{l}\text { Age at enrollment in } \\
\text { years. Median (range) }\end{array}$ & $\begin{array}{l}\text { Children*/adults } \\
\text { at enrollment }\end{array}$ & Western Europe & Eastern Europe & $\begin{array}{l}\text { Eastern - southern } \\
\text { Mediterrean }\end{array}$ & Others \\
\hline FMF & 554 & $268 / 286$ & $11(1-86)$ & $456 / 98$ & 297 & 4 & 214 & 39 \\
\hline TRAPS & 199 & $101 / 98$ & $23(2-77)$ & $85 / 114$ & 192 & 4 & 2 & 1 \\
\hline CAPS & 152 & $79 / 73$ & $15(0-77)$ & $91 / 61$ & 131 & 4 & 3 & 14 \\
\hline MKD & 104 & $47 / 57$ & $14(1-60)$ & $65 / 39$ & 94 & 4 & 1 & 5 \\
\hline Blau & 25 & $13 / 12$ & $13(3-51)$ & $18 / 7$ & 15 & 0 & 0 & 10 \\
\hline PAPA & 7 & $3 / 4$ & $18(4-30)$ & $3 / 4$ & 6 & 1 & 0 & 0 \\
\hline FCAS2 & 6 & $5 / 1$ & $50(13-79)$ & $2 / 4$ & 4 & 0 & 0 & 2 \\
\hline DIRA & 2 & $0 / 2$ & $5(1-9)$ & $2 / 0$ & 2 & 0 & 0 & 0 \\
\hline CRMO & 245 & $88 / 157$ & $14(1-61)$ & $204 / 41$ & 228 & 4 & 0 & 13 \\
\hline PFAPA & 361 & $208 / 153$ & $6(0-37)$ & $352 / 9$ & 221 & 66 & 69 & 5 \\
\hline Behçet & 65 & $27 / 38$ & $15(5-25)$ & $47 / 18$ & 52 & 7 & 4 & 2 \\
\hline Undefined PF & 160 & $77 / 83$ & $14(2-71)$ & $99 / 61$ & 146 & 12 & 1 & 1 \\
\hline
\end{tabular}

*age $<18$ years.

CAPS, diseases from the spectrum of cryopyrin associated periodic fever syndromes; CRM0, chronic recurrent multifocal osteomyelitis; DIRA, deficiency of the interleukin-1-receptor antagonist; F, female; FCAS2, familial cold autoinflammatory syndrome 2; FMF, familial Mediterranean fever; M, male; MKD, mevalonate-kinase deficiency; PAPA, pyogenic sterile arthritis, pyoderma gangrenosum and acne syndrome; PF, periodic fever; PFAPA, periodic fever syndrome with aphthous stomatitis, pharyngitis and cervical adenopathy; TRAPS,

tumour necrosis factor receptor associated periodic syndrome.

\section{METHODS}

\section{A common registry for autoinflammatory diseases}

The monogenic autoinflammatory diseases included were: FMF, CAPS, tumor necrosis factor -receptor associated periodic syndrome (TRAPS), Mevalonate kinase deficiency (MKD), Blau syndrome, Pyogenic Sterile Arthritis, Pyoderma Gangrenosum and Acne (PAPA) syndrome, DIRA and FCAS2. Other multifactorial autoinflammatory syndromes, such as chronic recurrent multifocal osteomyelitis (CRMO), Behcet's disease, periodic fever, aphthous stomatitis, pharyngitis and cervical adenitis (PFAPA) and patients with undefined periodic fevers were included.

Experts in autoinflammatory diseases and syndromes were initially asked to identify the variables they considered of interest for each disease. A draft data collection form was then sent to all the experts for their review. Five main categories were considered: (i) baseline information, (ii) clinical manifestations, (iii) laboratory examinations, (iv) imaging and other diagnostic procedures and (v) response to treatments. Further revisions of the forms were subsequently evaluated by the experts with a final approval of the definitive version by nominal group technique ${ }^{10}$ during a Consensus Meeting in March 2009.

The baseline data included: age, gender, country of birth and of residence, ethnicity, age at disease onset and at diagnosis and date of first and last visits to the treating center. Genetic testing was not mandatory for inclusion. Where performed, details on the genetic analysis were sought: gene screened, extent of the analysis (specific mutations, selected exons, complete gene sequencing), mutations detected (a list was continuously updated from the Infever database, http://fmf.igh.cnrs.fr/ISSAID/infevers//11, and laboratory where the analyses were performed. Finally, information on consanguinity and any relevant family history was collected. Patient data was anonymised and cases identified by alphanumeric codes. 


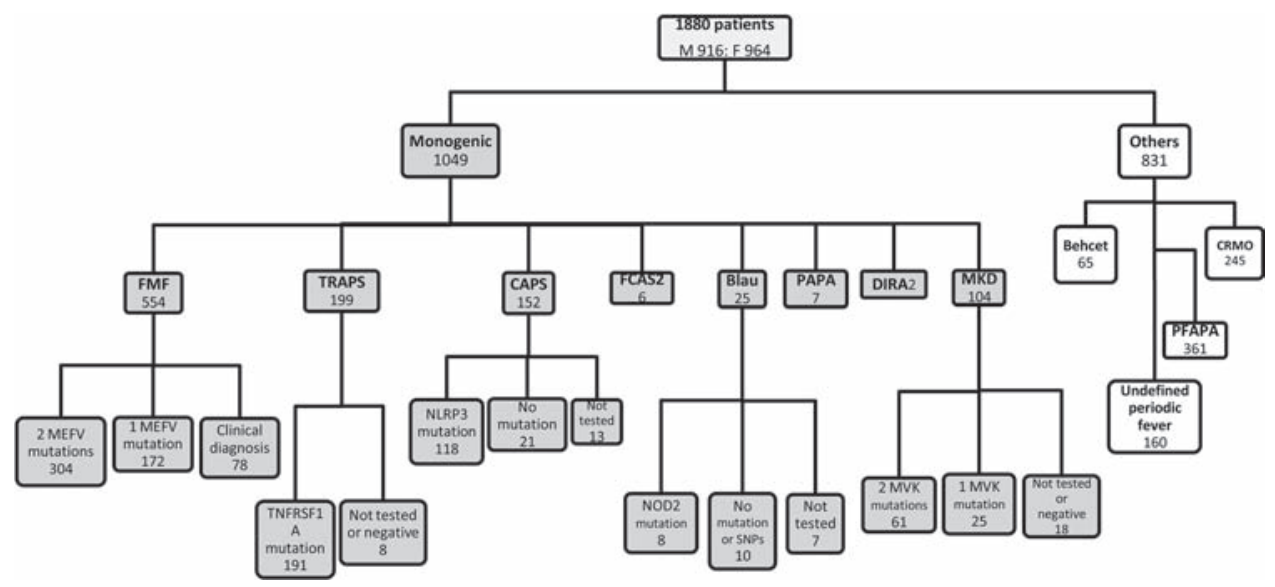

Figure 2 Distribution of patients with autoinflammatory diseases according to disease diagnosis and genetic confirmation. FMF, familial Mediterranean fever; MKD, mevalonate-kinase deficiency; TRAPS, tumour necrosis factor receptor associated periodic syndrome; CAPS, cryopyrin associated periodic syndromes; PAPA, pyogenic sterile arthritis, pyoderma gangrenosum and acne syndrome; DIRA, deficiency of the interleukin-1receptor antagonist; FCAS2, familial cold autoinflammatory syndrome 2; PFAPA, periodic fever, aphthous stomatitis, pharyngitis and cervical adenitis syndrome; CRMO, chronic recurrent multifocal osteomyelitis. MEFV, MEditerrean FeVer; TNFRSF1A, Tumour necrosis factor receptor superfamily member 1A; NOD2, nucleotide-binding oligomerisation domain-containing protein 2; NLRP3, NOD-like receptor 3; MVK, mevalonate-kinase; SNPs, single nucleotide polymorphisms.

\section{Dissemination and data collection}

The Eurofever project survey involved all centers linked to the Pediatric Rheumatology INternational Trial Organisation (PRINTO), a non-profit organisation devoted collaborative studies, that now encompass more than 400 centers in 60 countries worldwide $e^{12}$ and asked about their experience in autoinflammatory diseases. The survey results are available on the Eurofever website (www.printo.it/eurofever).

In addition, centers involved in the diagnosis and management of autoinflammatory diseases in adults were contacted and information on the study and how to participate was disseminated to members of the International Society of Systemic Autoinflammatory Diseases, the European League Against Rheumatism, the European Society of Immune-Deficiencies (ESID), through personal contacts, lectures and scientific communications and links to the Eurofever website.

The database has been developed with a sql server with through access via the member area of the PRINTO website (www.printo.it) with username and password on an https platform and URL parameters crypted. Only centers authorised (eg, centers with ethics committee approval) by the PRINTO coordinating were allowed to access the online database. Inclusion criteria for each disease were approved during the consensus conference and are reported in supplemental material. Ethical committee approval for entering patients in the registry was obtained in the participating centers as required by local legal requirements. ${ }^{12}$ Informed consent/assent as appropriate by the national laws in each country was signed by parents or legal representatives or child of adequate age. Enrollment started on November 2009.

Statistical tests were performed using subroutines from the statistical analysis package by MS Excel version 11.0.

\section{RESULTS}

\section{General demographic data}

By data lock in July 2011, 1880 patients (M:F=916:964) from 67 centers in 31 countries have been entered in the registry, 30 centers $(45 \%)$ required ethical committee approval. Enrolling countries, in alphabetic order, were: Albania, Argentina, Australia, Belgium, Croatia, Czech Republic, Denmark, France, Georgia,
Germany, Greece, Hungary, India, Israel, Italy, Latvia, Lithuania, Netherlands, Oman, Poland, Romania, Russia, Saudi Arabia, Serbia, Slovakia, Slovenia, Spain, Sweden, Switzerland, Turkey and the UK.

The same Centres enrolled also patients living in other countries (Algeria, Cyprus, Egypt, Guadalupe, Iceland, Ireland, Kuwait, Lebanon, Martinique, Morocco, Norway, Uruguay, Tunisia). The distribution of the patients by country of residence is shown in figure 1. Most of the enrolled patients (1388; 74\%) were resident in western Europe, 294 patients (16\%) in the eastern and southern Mediterranean (Turkey, Israel, north Africa), $106(6 \%)$ in eastern Europe (Croatia, Czech Republic, Hungary, Latvia, Lithuania, Poland, Romania, Russia, Serbia, Slovakia, Slovenia), 54 in Asia, 27 in South America and 11 in Australia. The number of enrolled patients for each disease and their distribution according to the region of residence are shown in table 1. At the time of enrollment, 1424 patients $(76 \%)$ were children and $456(24 \%)$ were adults.

\section{Monogenic autoinflammatory diseases}

In total, 1049 patients were enrolled with a diagnosis of a monogenic autoinflammatory disease; 703 had genetic results completely consistent with the diagnosis; 197 had a heterozygous mutation in a classically autosomal recessive disease; 93 had no mutations detected and 56 patients had not been genetically tested. Most of these latter patients were not resident in western Europe and testing was not performed for technical or financial reasons. The distribution of these patients is shown in figure 2. Conceivably, the rather high rate of genetically confirmed patients was due to the selective recruitment of these patients by the centers.

There was a marked delay between disease onset and diagnosis for all diseases - with a median of 7.3 years (figure $3 \mathrm{~A}$ ). Encouragingly there was a trend towards shorter diagnostic delay in patients born more recently (figure $3 \mathrm{~B}$ ). This was more obvious for diseases described over the last 3 or 4 decades, such as MKD, TRAPS and the more severe variant of CAPS known as CINCA/NOMID, especially after the respective molecular defects were identified. ${ }^{13-15}$ Nonetheless even in patients born in the 21st century the median diagnostic delay remained 
A

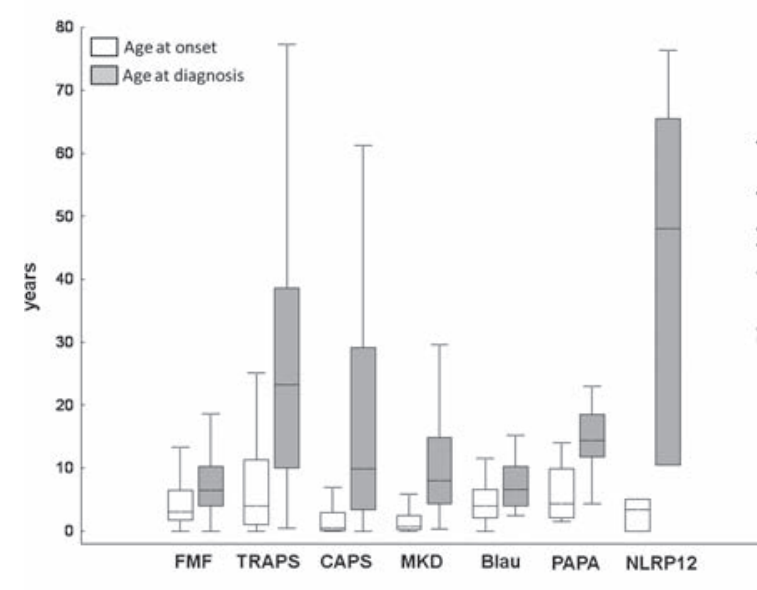

B

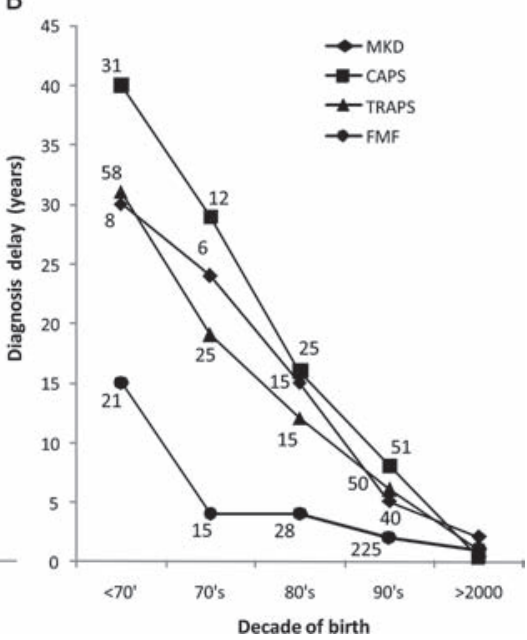

Figure 3 Diagnostic delay in patients with monogenic autoinflammatory diseases. The difference between the age at disease onset (white bars) and diagnosis (grey bars) is shown in panel A. Boxes contain values falling between the 25th and 75th percentiles, lines represent median values, whiskers lines that extend from the boxes represent the highest and lowest non-outlier values for each subgroups. Panel B: variation of diagnostic delay according to the decade of birth in four main inherited autoinflammatory diseases (FMF, familial Mediterranean fever; MKD, mevalonate-kinase deficiency; TRAPS, tumour necrosis factor receptor associated periodic syndrome CAPS, cryopyrin associated periodic syndrome). The number of patients are reported for each disease. For the first decade of the XXI century data on 265 FMF, 43 CAPS, 41 TRAPS and 35 MKD patients are available.

relatively long: 1.4 years (range $0.2-9$ ) for FMF, 1.2 years (0.1-7.4) for TRAPS, 1.4 years $(0.2-8.9)$ for CAPS and 2.5 years $(0.1-8.3)$ for MKD.

At the time of the enrollment the majority of patients (85\%) were still followed by the referral centers; 638/723 (89\%) children (age $<18$ years) and 254/326 (78\%) adults.

The distribution of the $554 \mathrm{FMF}$ patients (456 children and 98 adults) according to their ethnicity and region of residence is given in table 2. Mutations found in FMF patients are reported in supplementary table I. Consanguinity was present in 78 families, and 187 patients (34\%) declared a positive family history.

Among the 199 TRAPS patients (85 children, 114 adults) there were 45 separate mutations, 3 of which were previously unreported (supplementary table I). Eighty-four (42\%) patients reported a family history of disease; most of these carried a cysteine or the p.T50M mutation. The low-penetrance p.R92O and p.P46L variants were reported in 75 and 7 patients, respectively (supplementary table I). The careful analysis of the clinical manifestations of this latter group of patients will help in the characterisation of the actual impact of this variant on the phenotype in comparison with mutations of the TNFRSF1A gene. ${ }^{16}$

One hundred fifty-two patients (91 children and 61 adults) with CAPS were enrolled in the registry. Among 30 NLRP3 mutations detected, 2 were previously unreported (supplementary table I). A positive family history was present in 73 (48\%) patients.

There were 104 MKD patients (65 children, 39 adults) with 29 $M V K$ variants, 4 of which were previously unreported (supplementary table I). Eight families were consanguineous and there was a positive family history in $27(26 \%)$ patients.

Among the 25 Blau syndrome patients 8 had genetic confirmation. Following mutations were reported: p.R334Q, ${ }^{2}$ p.R334W, ${ }^{2}$ p.R587C. ${ }^{2}$ A new mutation, p.E600K, was found in 2 patients. Three polymorphisms were also found: p.P268S/SNP5 (five patients), p.R587R/SNP7 (one patient), p.R702W/SNP8 (one patient).

Six of seven patients with PAPA syndrome had confirmed mutations there were 3 previously described PSPTTP1 variants
(p.A230T, p.E250K and p.E250Q) and 3 novel variants (p.V344I, p.E256G, p.G904A) non already reported in Infevers.

There were six patients with the FCAS2 associated to NLRP12 mutations; a pair of twins from Guadalupe displayed the p.R284X mutation and four Italian patients displayed the p.D294E mutation.

Finally, two patients with DIRA were enrolled from the Netherlands and were homozygous for the E77X mutation of the IL1RN gene.

\section{Multifactorial conditions without clear genetic aetiology and undefined periodic fevers}

Two hundred forty-five patients (204 children and 41 adults) with CRMO were enrolled in the registry. The oldest patient was a 61-years-old man who was diagnosed in 2010. Six families were consanguineous and there was a positive family history in five patients.

Sixty-five patients with Behçet disease (47 children and 18 adults) were in the registry. Three families were consanguineous and there was a positive family history in 14 patients.

Three hundred sixty-one PFAPA patients were entered in the registry. Although nine of them were adults, the majority of patients $(88 \%)$ were less than 5 -years-old at disease onset, eight patients presented after the age of 10 . Five families were consanguineous and 36 other patients $(10 \%)$ presented with a family history of PFAPA. One hundred sixty patients $(99$ children and 61 adults) were enrolled in the category of undefined periodic fever. In the majority $(115,72 \%)$, the disease onset was in childhood. Twenty-four patients $(15 \%)$ presented after the age of 30 . Three families are consanguineous and a positive family history was reported in 14 sporadic cases. One hundred sixteen patients $(72 \%)$ were screened for at least one autoinflammatory diseases-associated gene. No mutations were found in $83 \%$. Notably, despite the presence of low-penetrance variants, 20 patients were included this subgroup as the entering centers felt that their clinical manifestations were not consistent with disease normally associated with that gene. The various mutations found were the p.R92Q variant of TNFRSF1A 
Table 2 Baseline data for familial Mediterranean fever patients

\begin{tabular}{|c|c|c|c|c|c|c|c|c|c|}
\hline & \multirow[b]{2}{*}{$\begin{array}{l}\text { Patient number } \\
(\%)\end{array}$} & \multirow[b]{2}{*}{$\mathbf{M} / \mathbf{F}$} & \multirow[b]{2}{*}{ Children/adults } & \multicolumn{6}{|c|}{ Ethnicity/country of residence } \\
\hline & & & & $\begin{array}{l}\text { Caucasianwestern } \\
\text { Europe }\end{array}$ & Turks in Turkey & Jews in Israel & $\begin{array}{l}\text { Arabst/Jews/ } \\
\text { Turks in WE* }\end{array}$ & Arabs $\neq$ & Other \\
\hline Genetically confirmed & $304(55)$ & $146 / 158$ & $249 / 55$ & 80 & 118 & 22 & $19 / 6 / 16$ & 24 & 19 \\
\hline Clinically suspected & $78(14)$ & $41 / 37$ & $59 / 19$ & 32 & 1 & 14 & $4 / 1 / 4$ & 14 & 8 \\
\hline All & 554 & $268 / 286$ & $456 / 98$ & 204 & 144 & 51 & $38 / 10 / 24$ & 47 & 36 \\
\hline
\end{tabular}

*resident in western Europe.

tincluding north Africans.

fincluding north Africans and Arabs living in north Africa and in Arabian countries.

F, female; $M$, male; WE, Western Europe.

(six patients); MEFV mutations in nine patients (p.R202Q, ${ }^{4}$ p.E148Q, ${ }^{2}$ p.A744S, ${ }^{1}$ p.S309F ${ }^{1}$ and p.V726A), ${ }^{1}$ the p.T356M mutation of $M V K$ (two patients), and the p.V198M, ${ }^{1}$ p. $Q 703 \mathrm{~K}^{1}$ and p.T219T ${ }^{1}$ variants of NLRP3.

\section{DISCUSSION}

To be successful, a rare disease registry needs to be genuinely international and requires active ongoing support by centers involved in diagnosing and managing the patients. PRINTO, which has a track record of establishing a successful network of centers in Pediatric Rheumatology, provided a starting point for this brand new initiative in the autoinflammatory diseases. Although this EU- funded project was particularly directed at Europe the pre-existing PRINTO network facilitated expansion to other geographic areas. The broad coverage of pediatric centers was crucial because, as shown by the present study, the majority of patients with autoinflammatory diseases have an early disease onset. ${ }^{17}$ Autoinflammatory diseases are also diagnosed by adult specialists (rheumatologists, clinical immunologists and geneticists, experts in amyloidosis) and other pediatric subspecialists (immunologists, neonatologists, experts in infectious diseases). A major effort of the project was to expand the network as widely as possible by actively inviting pediatric and adult centers known to have an interest in the diagnosis and management of these patients. This was made simpler by the involvement of initiatives such as EUROTRAPS (http://fmf.igh.cnrs.fr/ISSAID/ EUROTRAPS/) and Hyper IgD registry (http://hids.net) and international scientific societies. The ability to integrate with pre-existing registries and resources such as Infevers ${ }^{11}$ shows the power of web-based initiatives in collecting, collating and distributing data in rare diseases and in promoting networks of interested centres which can provide mutual support in diagnosis and management. Plans for further collaborations with other international and national initiatives are ongoing. ${ }^{18}$ Although a number of important geographic areas (North America, many south American and Asian countries) are not yet covered by the registry, the large number of patients recruited in this first period of the project and the wide geographical distribution is very encouraging and suggests that the network can continue to grow and prove an invaluable long term resource in the study of autoinflammatory diseases.

One of the most pressing issues in the rare diseases is delayed diagnosis. Autoinflammatory attacks are extremely unpleasant and frequently preventable with current treatments and untreated disease carries a risk of increased morbidity and irreversible long term damage. The dramatic decrease in diagnostic delay in the last decade is, to some extent, simply due to the fact that many of these diseases are recently recognised clinical entities and that genetic diagnosis has only become relatively widely available in the last few years. The first gene to be identified in autoinflammatory diseases was $M E F V$ in $1997^{2} 3$ and with identification of ILR $1 N$ in $2009^{7}$ the number of genetically characterised autoinflammatory diseases reached eight. Nonetheless the diagnosis of autoinflammatory diseases relies on a clinical suspicion and many cases are missed due to poor recognition of these exceptionally rare. The uneven geographic distribution of cases reported here with a marked bias towards western Europe, where there are a relatively large number of specialist centers with facilities for genetic analysis, highlights this problem and supports the results of a survey performed among PRINTO and ESID centers which suggested that poor recognition of the autoinflammatory diseases is even more marked in the eastern European countries. ${ }^{19}$

The main aim of our project was to set-up a long term registry for the very rare monogenic diseases (TRAPS, CAPS, MKD, Blau disease, NLRP12-associated disease, DIRA, PAPA syndrome). FMF has a high prevalence in some ethnicities and a previous multi-national collaboration (Meta-FMF) has collected a large case series. ${ }^{20}$ The aim of Eurofever in FMF was not to set up a formal registry of all existing FMF patients, but rather to collect information on unselected cohorts of FMF patients of different ethnicities and residing in different regions with a view to compare them with the other monogenic periodic syndromes. A cohort of patients with multifactorial diseases which enter the differential diagnosis, such as PFAPA syndrome, CRMO and Behçet disease, were also enrolled with a view to comparative studies.

Detailed data on clinical manifestations and response to treatment are already available for more than $70 \%$ of patients. For each completed patient, an internal validation process is provided by the participating experts. The aim of this validation process is to select patients with an unequivocal genetic and/or clinical picture for future studies on genotype-phenotype correlations, complication and response to treatment. Moreover, appropriate genetic testing will be offered to patients with a strong clinical suspicion for a given disease that has not been analysed so far. The registry is still actively recruiting and new patients will be included in the follow-up of the project that will continue in the long term. Moreover, newly recognised monogenic autoinflammatory diseases will be also included. ${ }^{21-23}$

Establishment of a large cohort and prolonged follow-up data will allow statistically robust and clinically meaningful analysis of phenotype/genotype correlations, complications and response to treatment for most of the disease involved. Standardised collection of clinical data on both rare monogenic autoinflammatory diseases and more frequent conditions will facilitate comparison of different diseases. This will overcome the problems inherent in single disease registries when generating evidence-based diagnostic and classification criteria and/or indications for the molecular analysis ${ }^{24} 25$. 
Approximately $75-80 \%$ of patients with clinical features consistent with autoinflammatory diseases have no recognised mutations in any of the known genes. ${ }^{24} 26-28$ A large cohort of patients with undefined periodic fevers will allow characterisation of novel genes in this currently challenging disease group. For each patient of this particular subgroup a panel of experts will evaluate the possible indications for the implementation of molecular screening for already known genes. In any case, the identification of 'genetically negative' patients with a well characterised clinical phenotype (ie, CAPS-like patients) and the cohort of individuals and families with an undefined periodic fever syndrome are a future resource for linkage and/or next generation sequencing analyses. ${ }^{29} 30$

Acknowledgements: The Authors would like to thank Eugenia Mosci for her outstanding and enthusiastic secretarial assistance and the webmaster Michele Pesce for his excellent work and continuous kind assistance.

Funding This project is supported by Executive Agency for Health and Consumers of European Union (EAHC, Project No2007332, Eurofever) and through Coordination Theme 1 (Health) of the European Community's FP7, Grant agreement number HEALTH-F2-2008-200923 (Eurotraps). Unrestricted educational grants were also kindly provided by PRINTO and Novartis.

Correction notice This article has been corrected since it was published Online First. The following statement was added at the end of the author list: for Paediatric Rheumatology International Trials Organisation (PRINTO), Eurotraps and Eurofever Projects.

\section{Competing interests None.}

Provenance and peer review Not commissioned; externally peer reviewed.

Author affiliations ${ }^{1}$ Department of Paediatrics, Division of Allergology, Rheumatology and Clinical Immunology, University Medical Centre Ljubljana, Ljubljana, Slovenia

2Department of Paediatrics, University Medical Center Utrecht, Utrecht, Netherlands ${ }^{3}$ Department of Pediatric Nephrology and Rheumatology, Hacettepe University Children's Hospital, Ankara, Turkey

${ }^{4}$ Division of Medicine, University College London Medical School, Royal Free Campus, London, UK

${ }^{5}$ Center of Paediatric and Adolescent Rheumatology - UCL, Great Ormond Street

Children Hospital, London, UK

${ }^{6}$ Pediatric rheumatology and CEREMAI, Bicêtre hospital, University of Paris Sud, Bicetre, France

${ }^{7}$ Pediatric Rheumatology Unit, Bambino Gesù Children's Hospital, Rome, Italy ${ }^{8}$ Department of Pediatric Immunology and Rheumatology, Necker Hospital, Paris, France

${ }^{9}$ Department of Paediatrics, Centre Hospitalier Universitaire Vaudois (CHUV),

Lausanne, and Hôpitaux Universitaires, Geneva, Switzerland

${ }^{10}$ Department of Pediatrics and Adolescent Medicine, Charles University in Prague and General University Hospital, Praha, Czech Republic

${ }^{11}$ Division of Pediatric Rheumatology, Department of Pediatrics, University Children's Hospital Tübingen, Tübingen, Germany

${ }^{12}$ Medical Unit for Autolnflammatory diseases, Hôpital Arnaud de Villeneuve,

Montpellier, France

${ }^{13}$ Departement of pediatrics, Hôpital Mignot, Le Chesnay, France

${ }^{14}$ Department of General Internal Medicine, Radboud University Nijmegen Medical

Center, Nijmegen, Netherlands

${ }^{15}$ Department of Pediatrics, Clinic for Pediatric and Adolescent Medicine, Vivantes

Klinikum im Friedrichshain, Berlin, Germany

${ }^{16}$ Divison of Rheumatology, DuPont Children's Hospital, Thomas Jefferson University,

Wilmington, Delaware, USA

${ }^{17}$ Department of Pediatrics, Division of Pediatric Rheumatology, University Hospital

Gasthuisberg, Leuven, Belgium

${ }^{18}$ Department of Paediatrics, Detska Fakultna Nemocnica, Kosice, Slovakia

${ }^{19}$ Immunology Department. CDB. Hospital Clínic/IDIBAPS, Barcelona, Spain

${ }^{20}$ Department of Infectious Diseases and Immunology, University Children's Hospital,

Munich, Germany

${ }^{21}$ Division of Rheumatology, Cerrahpasa Medical Faculty Medicine, Istanbul, Turkey

${ }^{22}$ Pediatria II, Reumatologia, IRCCS Istituto G Gaslini, Università di Genova, Genova, Italy

${ }^{23}$ Pediatria II, Reumatologia, IRCCS Istituto G Gaslini, Genova, Italy

\section{REFERENCES}

1. Masters SL, Simon A, Aksentijevich I, et al. Horror autoinflammaticus: the molecular pathophysiology of autoinflammatory disease $\left({ }^{*}\right)$. Annu Rev Immunol 2009:27:621-68
2. The French FMF Consortium. A candidate gene for familial Mediterranean fever. Nature Genetics 1997;17:25-31.

3. The International FMF Consortium. Ancient missense mutations in a new member of the RoRet gene family are likely to cause familial Mediterranean fever. Cell 1997:90:797-807.

4. McGonagle D, McDermott MF. A proposed classification of the immunological diseases. PLoS Med 2006; 3:e297.

5. Ben-Chetrit E, Touitou I. Familial mediterranean Fever in the world. Arthritis Rheum 2009;61:1447-53.

6. Cuisset L, Jeru I, Dumont B, et al. Mutations in the autoinflammatory cryopyrinassociated periodic syndrome gene: epidemiological study and lessons from eight years of genetic analysis in France. Ann Rheum Dis 2011;70:495-9.

7. Aksentijevich I, Masters SL, Ferguson PJ, et al. An autoinflammatory disease with deficiency of the interleukin-1-receptor antagonist. N Engl J Med 2009; 360:2426-37

8. Jéru I, Duquesnoy P, Fernandes-Alnemri T, et al. Mutations in NALP12 cause hereditary periodic fever syndromes. Proc Natl Acad Sci USA 2008;105:1614-9.

9. Ozen S, Frenkel J, Ruperto N, et al. The Eurofever Project: towards better care for autoinflammatory diseases. Eur J Pediatr 2011;170:445-52.

10. Ruperto N, Meiorin S, lusan SM, et al. Consensus procedures and their role in pediatric rheumatology. Curr Rheumatol Rep 2008;10:142-6.

11. Milhavet $\mathbf{F}$, Cuisset L, Hoffman HM, et al. The infevers autoinflammatory mutation online registry: update with new genes and functions. Hum Mutat 2008;29:803-8.

12. Ruperto N, Martini A. Networking in paediatrics: the example of the Paediatric Rheumatology International Trials Organisation (PRINTO). Arch Dis Child 2011:96:596-601.

13. Drenth JP, Cuisset L, Grateau G, et al. Mutations in the gene encoding mevalonate kinase cause hyper-lgD and periodic fever syndrome. International Hyper-lgD Study Group. Nat Genet 1999;22:178-81.

14. Houten SM, Kuis W, Duran M, et al. Mutations in MVK, encoding mevalonate kinase, cause hyperimmunoglobulinaemia D and periodic fever syndrome. Nat Genet 1999;22:175-7.

15. McDermott MF, Aksentijevich I, Galon J, et al. Germline mutations in the extracellular domains of the $55 \mathrm{kDa}$ TNF receptor, TNFR1, define a family of dominantly inherited autoinflammatory syndromes. Cell 1999;97:133-44.

16. Pelagatti MA, Meini A, Caorsi R, et al. Long-term clinical profile of children with the low-penetrance R920 mutation of the TNFRSF1A gene. Arthritis Rheum 2011:63:1141-50.

17. Gattorno M, Federici S, Pelagatti MA, et al. Diagnosis and management of autoinflammatory diseases in childhood. J Clin Immunol 2008;28 Suppl 1:S73-83

18. Lainka E, Bielak M, Hilger V, et al. Translational research network and patient registry for auto-inflammatory diseases. Rheumatology (Oxford) 2011;50:237-42.

19. Toplak N , Dolezalovà $P$, Constantin $T$, et al. Periodic fever syndromes in Eastern and Central European countries: results of a pediatric multinational survey. Pediatr Rheumatol Online J 2010;8:29.

20. Touitou I, Sarkisian T, Medlej-Hashim M, et al. Country as the primary risk factor for renal amyloidosis in familial Mediterranean fever. Arthritis Rheum 2007:56:1706-12.

21. Liu $\mathbf{Y}$, Ramot $Y$, Torrelo A, et al. Mutations in PSMB8 cause CANDLE syndrome with evidence of genetic and phenotypic heterogeneity. Arthritis Rheum. Published Online First: 27 Sept 2011. doi:10.1002/art

22. Agarwal AK, Xing C, DeMartino GN, et al. PSMB8 encoding the 35 proteasome subunit is mutated in joint contractures, muscle atrophy, microcytic anemia, and panniculitis-induced lipodystrophy syndrome. Am J Hum Genet 2010;87:866-72

23. Marrakchi S, Guigue P, Renshaw BR, et al. Interleukin-36-receptor antagonist deficiency and generalized pustular psoriasis. N Engl J Med 2011;365:620-8.

24. Gattorno M, Sormani MP, D'Osualdo A, et al. A diagnostic score for molecular analysis of hereditary autoinflammatory syndromes with periodic fever in children. Arthritis Rheum 2008;58:1823-32.

25. Touitou I, Hentgen V, Koné-Paut I. Web resources for rare auto-inflammatory diseases: towards a common patient registry. Rheumatology (Oxford) 2009;48:665-9.

26. Simon A, van der Meer JW, Vesely R, et al. Approach to genetic analysis in the diagnosis of hereditary autoinflammatory syndromes. Rheumatology (Oxford) 2006; $45: 269-73$

27. Federici L, Rittore-Domingo C, Koné-Paut I, et al. A decision tree for genetic diagnosis of hereditary periodic fever in unselected patients. Ann Rheum Dis 2006;65:1427-32.

28. Tchernitchko $\mathbf{D}$, Moutereau S, Legendre M, et al. MEFV analysis is of particularly weak diagnostic value for recurrent fevers in Western European Caucasian patients. Arthritis Rheum 2005:52:3603-5.

29. $\mathbf{N g} \mathbf{S B}$, Buckingham KJ, Lee C, et al. Exome sequencing identifies the cause of a mendelian disorder. Nat Genet 2010;42:30-5.

30. Ku CS, Naidoo N, Pawitan Y. Revisiting Mendelian disorders through exome sequencing. Hum Genet 2011;129:351-70. 


\section{$\Delta$ An International registry on Autoinflammatory diseases: the Eurofever experience}

Natasa Toplak, Joost Frenkel, Seza Ozen, Helen J Lachmann, Patricia Woo, Isabelle Koné-Paut, Fabrizio De Benedetti, Benedicte Neven, Michael Hofer, Pavla Dolezalova, Jasmin Kümmerle-Deschner, Isabelle Touitou, Veronique Hentgen, Anna Simon, Herman Girschick, Carlos Rose, Carine Wouters, Richard Vesely, Juan Arostegui, Silvia Stojanov, Huri Ozgodan, Alberto Martini, Nicolino Ruperto, Marco Gattorno and for the Paediatric Rheumatology International Trials Organisation (PRINTO), Eurotraps and Eurofever Projects

Ann Rheum Dis 2012 71: 1177-1182 originally published online February 29, 2012

doi: 10.1136/annrheumdis-2011-200549

Updated information and services can be found at:

http://ard.bmj.com/content/71/7/1177

\section{These include:}

References This article cites 29 articles, 7 of which you can access for free at: http://ard.bmj.com/content/71/7/1177\#BIBL

Email alerting Receive free email alerts when new articles cite this article. Sign up in the service box at the top right corner of the online article.

Topic Articles on similar topics can be found in the following collections Collections Genetics (844)

\section{Notes}

To request permissions go to:

http://group.bmj.com/group/rights-licensing/permissions

To order reprints go to:

http://journals.bmj.com/cgi/reprintform

To subscribe to BMJ go to:

http://group.bmj.com/subscribe/ 